\title{
Pulmonary rehabilitation in interstitial lung diseases: A review of the literature
}

\author{
Krzysztof Wytrychowski ${ }^{1, A-D, F}$, Anna Hans-Wytrychowska ${ }^{2, A, B, D, E}$, Paweł Piesiak ${ }^{3, B, C, E}$, \\ Marta Majewska-Pulsakowska ${ }^{4, A-E}$, Krystyna Rożek-Piechura ${ }^{5, E, F}$ \\ 1 Department and Clinic of Internal Medicine, Pneumonology and Allergology, Wroclaw Medical University, Poland \\ ${ }^{2}$ Department of Family Medicine, Wroclaw Medical University, Poland \\ ${ }^{3}$ Department and Clinic of Pulmonology and Lung Cancers, Wroclaw Medical University, Poland \\ ${ }^{4}$ Department and Division of Medical Rehabilitation, Wroclaw Medical University, Poland \\ ${ }^{5}$ Department of Rehabilitation in Internal Diseases, University School of Physical Education, Wrocław, Poland \\ A - research concept and design; B - collection and/or assembly of data; C - data analysis and interpretation; \\ $D$ - writing the article; $E$ - critical revision of the article; $F$ - final approval of the article
}

Address for correspondence

Krzysztof Wytrychowski

E-mail:anhw@op.pl

\section{Funding sources}

None declared

\section{Conflict of interest}

None declared

Received on August 24, 2018

Reviewed on December 2, 2018

Accepted on December 12, 2019

Published online on February 26, 2020

Cite as

Wytrychowski K, Hans-Wytrychowska A, Piesiak P, MajewskaPulsakowska M, Rożek-Piechura K. Pulmonary rehabilitation in interstitial lung diseases: A review of the literature. Adv Clin Exp Med. 2020;29(2):257-264.

doi:10.17219/acem/115238

DOI

10.17219/acem/115238

\section{Copyright}

Copyright by Author(s)

This is an article distributed under the terms of the

Creative Commons Attribution 3.0 Unported (CC BY 3.0)

(https://creativecommons.org/licenses/by/3.0/)

\begin{abstract}
There are more than 200 different diseases classed as interstitial lung diseases (ILDs). For epidemiological and practical purposes, ILDs are classified into diseases of known and unknown etiology. The aim of this review is to evaluate our current knowledge about the efficacy and safety of pulmonary rehabilitation (PR) in patients with ILDs. Other issues, such as ILD pathogenesis, prevalence and comorbidity, are also elaborated in the review. Pulmonary rehabilitation is an important part of comprehensive care for patients with ILDs. In comparison to PR for patients with chronic pulmonary obstructive disease (COPD), the number of clinical studies concerning PR for patients with ILDs is small. The majority of trials have been performed in relatively small groups of patients. The principles of PR in this group of patients are the same as for patients with COPD. Exercise-induced desaturation is frequently observed during $P R$, which is the main source of complications in patients with ILDs. Major differences between ILD and COPD patients include poorer exercise tolerance and faster development of respiratory failure in patients with ILDs.
\end{abstract}

Key words: pulmonary rehabilitation, airway management, interstitial lung diseases 


\section{The pathogenesis and prevalence of interstitial lung diseases}

Interstitial lung diseases (ILDs) constitute a heterogeneous group of about 200 diseases characterized by acute or chronic follicular and bronchial inflammation and progressive, usually irreversible, pulmonary fibrosis, bilateral diffuse pulmonary lesions in imaging examinations, and ventilation restrictions. Despite the differentiated etiology, the clinical image of ILDs is similar, and results from the presence of diffuse and irreversible fibrous lesions of the alveolar parenchyma of the lung interstitium. Specific forms of ILDs can be differentiated from one another based on clinical data, radiological imaging and lung biopsy. The histopathologic changes in the lungs of patients with ILDs can range from granulomatous inflammation without parenchymal fibrosis in patients with sarcoidosis to expansive pulmonary fibrosis with architectural distortion of the lung in patients with idiopathic pulmonary fibrosis (IPF). Clinical ILD features include exercise-induced dyspnea, exercise-induced hypoxemia, progressive skeletal muscle weakness, and deterioration of exercise tolerance. The main symptoms of fibrosis are progressive decreases in forced vital capacity (FVC) and in the diffusing capacity of the lung for carbon monoxide (DLCO). Restriction of pulmonary ventilation leads to respiratory failure, further enhanced by diminished alveolar blood flow resulting from capillary destruction and hypoxic vasoconstriction. ${ }^{1}$ The onset of the disease is usually non-specific; patients may complain of a low-grade fever or a fever with unclear etiology, coughing and deteriorated exercise tolerance. If the patient is a smoker, chronic pulmonary obstructive disease (COPD) is usually suspected, because spirometry focusses on disorders in lung ventilation. In patients with ILD, airflow in the respiratory tract is not disturbed; obstruction is rare, except in patients with sarcoidosis. ${ }^{2} \mathrm{~A} \mathrm{di-}$ agnosis of ILD is sometimes based on changes in the chest $\mathrm{X}$-ray image, especially in patients in whom this examination is performed periodically due to occupational exposure. In some cases, the onset of ILD may be acute, with symptoms requiring immediate medical intervention: pneumothorax, hemoptysis and rapid progressive respiratory failure. Interstitial lung diseases may be associated with exposure to occupational and environmental factors, drugs (amiodarone, chemotherapy drugs, methotrexate), or be accompanied by multiple connective tissue diseases (systemic lupus erythematosus, rheumatoid arthritis, systemic scleroderma, or dermatomyositis). In many cases, the etiology is unknown.

In a European study the prevalence of ILDs was estimated of 97.9/10,000. The most prevalent diagnoses were sarcoidosis (42.6\%), connective tissue disease-associated ILDs (CTD-ILDs) (16\%), IPF (11.6\%), and occupational ILDs $(5.0 \%){ }^{3}$ The data from earlier studies also showed that the most frequent ILDs are IPF and sarcoidosis, which together comprise about $50 \%{ }^{4}$

\section{Management}

Due to the different pathogenic mechanisms, treatment for ILDs varies depending on the diagnosis. In the case of a known cause, such as for example exposure to environmental agents or drugs, the primary treatment is to eliminate the factors causing the disease. If the disease has an autoimmune background, glucocorticoids and immunosuppressive drugs are used to reduce the inflammation leading to progressive fibrosis. However, in some diseases of unknown etiology, such as IPF, there are still no effective pharmacological methods that significantly improve survival. Intensive research has been carried out in the past decade to find effective antifibrotic and anti-inflammatory drugs. ${ }^{5}$

Thus ILD treatment often includes only oxygen therapy in case of respiratory failure. The progression of lung fibrosis depends on the underlying disease, and is particularly rapid in IPF. In selected patients with treatment-resistant progressive ILD, the only therapeutic method is lung transplantation. However, due to an insufficient number of donors and numerous contraindications, access to this method is limited. Therapeutic options for ILDs are often limited, and do not guarantee improvement in the quality of life or its prolongation. ${ }^{5,6}$ An increasing number of reports indicate that in most patients with ILDs, pulmonary rehabilitation (PR) is a widely accessible treatment that can significantly improve exercise capacity and reduce dyspnea. ${ }^{7}$

\section{Pulmonary rehabilitation principles}

Pulmonary rehabilitation is an evidence-based, multidisciplinary and comprehensive medical intervention dedicated to patients with a variety of symptoms of chronic diseases and reduced daily activity. The combination of PR with an individualized treatment plan reduces the symptoms, improves physical fitness, enhancing the functional and psychological status and quality of life of patients with respiratory diseases. The primary problem of patients with progressive chronic lung diseases is a gradual increase in symptoms such as dyspnea, persistent cough, easy fatigue and weakness, which cause restrictions of daily physical activity, resulting in muscle weakness and functional limitations. The patient becomes disabled, often experiences anxiety and/or depression and gradually withdraws from social life. The quality of life deteriorates considerably.

The purposes of PR include physical capacity improvement, reduction in disease symptoms, relief from negative emotional states, development of a healthy lifestyle with adequate physical activity, elimination of smoking, and giving patients the ability to self-monitor their symptoms, the course of the disease and their compliance with recommended treatments. Pulmonary rehabilitation includes physical examinations, chest physiotherapy, individual strength, 
endurance and respiratory training, as well as relaxation techniques aimed at reducing muscle tension and anxiety and/or depression. Patients are educated about the disease, lifestyle, nutrition, and the harmfulness of smoking.

Pulmonary rehabilitation has been used for many years in patients with COPD, and numerous benefits have been noted, particularly reduced dyspnea, increased effort tolerance and improved quality of life. ${ }^{7}$ The indications for PR and its benefits for patients with COPD have been well-defined. ${ }^{8}$

According to American Thoracic Society/European Respiratory Society guidelines, rehabilitation should be performed by a multidisciplinary team of pulmonologists, physiotherapists, psychologists, and nurses. ${ }^{7}$ The first step is detailed diagnostic testing that allows the PR to be adjusted to the current state of the patient, including an evaluation of the patient's quality of life, the degree of anxiety and depression, and an evaluation of minimal exercise tolerance. In addition, the strength of the peripheral muscles can be assessed. A six-minute walk test (6MWT) is a commonly used method of evaluating exercise tolerance. It is extremely important to evaluate $\mathrm{O}_{2}$ saturation during exercise, and in particular the greatest decrease in $\mathrm{O}_{2}$ saturation during the $6 \mathrm{MWT}$ (nadir $\mathrm{SPO}_{2}$ ). ${ }^{8}$

Pulmonary rehabilitation includes both education and supervised exercise. The scope of the education should include an explanation of the pathomechanisms of the disease, an explanation of the appropriate treatment, breathing techniques, principles of healthy nutrition, indications for air travel in patients with respiratory failure, the correct use of inhalers, and dealing with stress. Physical exercises include individually modified endurance and aerobic training.

The main problems hindering the widespread use of PR are limited funding from healthcare systems despite increasing awareness of the benefits of PR, a lack of knowledge among physicians and a shortage of experienced rehabilitators. Pulmonary rehabilitation should be standard care in patients with COPD, along with pharmacotherapy, oxygen therapy and non-invasive ventilation.

Pulmonary rehabilitation favorably modifies the course of COPD in many ways, causing:

1. reduced number of hospitalizations,

2. increased exercise tolerance,

3. reduced dyspnea,

4. increased resistance and muscle strength,

5. improved quality of life,

6. reduced emotional disorders,

7. increased self-reliance,

8. increased fat-free body mass,

9. increased breath volume and oxygen saturation, and

10. improved capacity of the skeletal muscles at the cellular level. 7

From a clinical point of view, the exercise component of PR increases the patient's physical capacity, which is the most often measured by the $6 \mathrm{MWT}$ or oxygen uptake during a maximal cardiopulmonary exercise test (maximal oxygen uptake - $\mathrm{VO}_{2} \mathrm{max}$ ). The obvious benefits of PR mean that over the years, attempts have been made to extend PR indications to other groups of patients with chronic lung diseases.

\section{Pulmonary rehabilitation in ILDs}

Although ILDs and COPD are completely different diseases, they are similar in many ways: Both diseases involve respiratory distress, increased respiratory effort and abnormal gas exchange, and the incidence of anxiety and depression is higher than in the general population. Numerous similarities indicate that PR in patients with ILDs may produce similar beneficial effects as in those with COPD. ${ }^{9}$ Among ILD patients, those with IPF have particularly poor prognoses. The median of survival since the diagnosis has not changed in this group in the last 30 years, and it is no more than 5 years. ${ }^{10}$ Until recently, apart from oxygen therapy, there was no therapeutic option for these patients. Because of the rapid progression of the disease, patients with IPF are rarely qualified for PR. The recent introduction of 2 new medicinal products for IPP - pirfenidone and the tyrosine kinase inhibitor nintedanib - may improve this situation. Early addition of PR to pharmacotherapy can improve the patients' survival. ${ }^{6}$

No guidelines concerning the optimization of exercise for patients with ILDs have been developed yet. Randomized trials have included all interstitial disorders or have been dedicated to a defined subgroup, such as patients with IPF. ${ }^{11,12}$ The results of PR results are highly affected by the diagnosis. This especially applies to IPF patients, who show less improvement in physical performance after PR compared to patients with other ILDs. However, the faster course of IPF compared to most ILDs should be taken into account. ${ }^{13}$

The basic parameter for PR performance evaluation is the change in minimal important difference (MID) in the 6MWT. The MID has been set at $54 \mathrm{~m}$ in people with COPD, although there are opinions that this value is exaggerated. ${ }^{14}$ For patients with ILDs, the MID value has not been established; the range varies from $24 \mathrm{~m}$ to $45 \mathrm{~m}$, depending on the statistical method chosen. ${ }^{15,16}$ The severity of symptoms, mainly dyspnea, is also evaluated. The Borg Dyspnea Scale and the Medical Research Council's (MRC) Baseline Dyspnea Index (BDI) and Transition Dyspnea Index (TDI) are used to evaluate the severity of dyspnea. Cough, depression, anxiety, and fatigue may also be evaluated. For the health-related quality of life evaluation, the most commonly used questionnaires are the Short Form Health Survey (SF-36), the Chronic Respiratory Disease Questionnaire (CRDQ) and St. George's Respiratory Questionnaire (SGRQ) ${ }^{17,18}$ An additional parameter is pulse oximetry during exercise to determine nadir $\mathrm{SpO}_{2}$ during the $6 \mathrm{MWT}{ }^{19}$ 
The number of controlled PR studies in ILDs is small, but a systematic increase is noticeable. A meta-analysis published in 2014 included a total of 8 papers concerning PR in patients with ILDs. No side effects were observed in any of the studies. The weighted mean difference in the distance in the 6MWT was $44.34 \mathrm{~m}$. Statistically significant reductions in dyspnea was observed in all the studies. Improved quality of life was identified using various questionnaires. The improvement directly after PR that was observed in the subpopulation of IPF patients was similar to that of the whole population examined. The limitation was the small number of study groups (in total, 86 patients underwent PR and 82 comprised the control groups). ${ }^{20}$ In a meta-analysis of 142 patients with IPF published in 2018, 4 studies evaluated the short-term benefits of PR. Significant improvement was found in the $6 \mathrm{MWT}$, along with a significant decrease in the total SGRQ score. ${ }^{21}$

In a large study including 402 patients on a widespread ILD spectrum, PR was performed at a specialist center for an average of 30 days with 5 sessions per week. Clinically significant improvements in the patients' quality of life, physical capacity (the mean increase compared to the baseline 6MWT was $46 \mathrm{~m}$ ) and a slight improvement in FVC $(+1 \%)$ were observed. The only predictor of a significant response to PR was a low baseline 6MWT; the largest increase in the distance was observed in this group. Low $6 \mathrm{MWT}$ scores occurred in patients with low FVC, low total lung capacity (TLC) and treated with home oxygen therapy. In this study, conducted in an inpatient setting, $80 \%$ of the patients were on home oxygen therapy. This is further evidence of the value of PR use in patients with advanced respiratory failure. ${ }^{22}$

Different parameters are evaluated in different publications. The manner in which PR is performed (outpatient or inpatient) also varies from study to study; the study groups include patients with assorted ILDs or only 1 disease, e.g., IPF, and the duration of PR varies. However, despite the lack of detailed guidelines, $\mathrm{PR}$ is becoming an increasingly common component of multidisciplinary care for people with ILDs.

It is currently believed that patients with ILDs should be included in PR programs typically lasting 8 weeks, with at least 2 weekly sessions of at least 30 min of aerobic training. Spirometry tests play a lesser role in qualifying ILD patients for PR than in case of COPD, because a small degree of dysfunction in lung function tests is often observed in patients with significant limitations to exercise tolerance. The mechanism of exercise dyspnea in ILDs is complex. The primary role is played by dysfunction of the alveolar-capillary barrier associated with thickening of the pulmonary artery walls. Developing pulmonary hypertension is a result of chronic hypoxia-induced vasoconstriction and thromboembolic changes leading to the destruction of blood vessels. There is a strong correlation between increases in pulmonary pressure and decreases in exercise tolerance. In addition, an impaired chronotropic response of the heart to exercise and prolonged tachycardia are often observed, which may indicate cardiac sarcoidosis. In some patients, an excessively fast resting heart rate may limit exercise capacity, since the heart rate prevents the patients from continuing exercise shortly after they begin. In this situation, the use of drugs with negative chronotropic effects can be beneficial in terms of the results of rehabilitation. However, the implementation of this kind of therapy in the population with pulmonary pathologies has numerous limitations, including the small group of available drugs. On the one hand, some of them, such as ivabradine, have not been tested in these patients; and on the other, $\beta$-blockers may have a potentially adverse effect. Nebivolol, a $\beta 1$-selective agent, is preferred in these patients because it causes quite low negative chronotropic effects at the standard dose used in hypertension compared to other representatives of the class. Another common problem is the coexistence of left ventricular diastolic dysfunction in many patients. In this situation, a lack of proper heart rate control impairs exercise tolerance, thus significantly affecting training opportunities.

Worsening tolerance to exercise may also be caused by skeletal muscle dysfunction. The mechanisms of this phenomenon include changes in skeletal muscles in the course of an underlying disease (e.g., dermatomyositis); myopathy induced by chronic systemic steroid therapy; and progressive immobilization of the patient. ${ }^{22}$

A progressive decrease in the 6MWT is a predictor of increased mortality, especially in IPF patients, among whom a drop in the $6 \mathrm{MWT}$ by more than $61 \mathrm{~m}$ in 6 months and desaturation below $88 \%$ during exercise are associated with increased mortality. ${ }^{8}$

The course and prognosis of ILDs is affected not only by the primary disease, but also by accompanying ones. Exercise tolerance in ILD patients is mainly affected by cardiovascular and metabolic diseases and smoking. ${ }^{23}$ Accompanying diseases are divided into pulmonary and extrapulmonary ones. Pulmonary fibrosis enhances the expression of cytokines and growth factors, promoting the development of atherothrombosis through an increase in systemic inflammation and hypercoagulability. This leads to a two-fold increase in the risk of ischemic heart disease (IHD) compared to the general population. The coexistence of pulmonary fibrosis and IHD significantly shortens the patients' survival time. ${ }^{20}$ Among IPF patients, as many as 1/3 have recognized IHD. The risk of venous thromboembolism is more than three-fold higher in the population of patients with ILDs compared to the general population. This requires early implementation of antithrombotic prophylaxis during hospitalization. ${ }^{24}$

Pulmonary arterial hypertension (PAH), defined as mean pulmonary arterial pressure $\geq 25 \mathrm{~mm} \mathrm{Hg}$ evaluated during right heart catheterization, may occur in any chronic lung disease. This also applies to patients with ILDs, among whom the risk of PAH depends on the diagnosis. 
It is generally accepted that the more severe the course of the disease is, the higher the incidence of PAH. For example, PAH is more common in patients with IPF than in cases of sarcoidosis. A diagnosis of PAH results in rapid deterioration of physical performance, the need for home oxygen therapy and increased mortality. Transthoracic echocardiography and right heart catheterization are routinely used in the diagnostics. The simple test is the $6 \mathrm{MWT}$, which reveals an impaired normalization of the heart rate after exercise: A heart rate difference immediately after exercise and after $1 \mathrm{~min}$ rest that is lower than 13 bpm suggests PAH. ${ }^{25}$ Special attention is required in case of patients with PAH in the phase of right-sided heart failure. The presence of clinically prominent PAH leads to recommendations for limiting intense exercise, with the fear that exercise-induced rapid pulmonary hypertension with the symptoms of right ventricular circulatory failure may lead to sudden death. ${ }^{26}$ Pulmonary arterial hypertension patients are at an increased risk of PR complications, including arrhythmias, syncope and dizziness, which may occur in $13 \%$ of patients. ${ }^{26,27}$

Physical exercise is associated with sympathetic activation, which increases the risk of arrhythmias. At the same time, the population of patients with respiratory diseases is thought to have hypoxia-related arrhythmias. In the light of some research, this is not a significant impediment to physical activity. Although the majority of patients had 24-hour asymptomatic single premature supraventricular and ventricular beats, no significant arrhythmia was observed during the $6 \mathrm{MWT}$ despite a significant decrease in $\mathrm{SpO}_{2} \cdot{ }^{27}$

The risk of complications during PR decreases when interval training with a maximum heart rate increase up to $120 \mathrm{bpm}$ and a combination of endurance and strength exercises is used. ${ }^{28}$

Gastroesophageal reflux disease (GERD) is a very common extrapulmonary comorbidity. In patients with IPF, the incidence is up to $80 \%$, and symptoms may persist despite the use of proton pump inhibitors. ${ }^{25}$ Sleep apnea syndrome occurs in more than half of the patients. Predictors are a high body mass index (BMI) and deteriorating lung function. ${ }^{24}$ Depression, diagnosed in approx. $25 \%$ of patients with ILDs, especially in the group with severe dyspnea, sleep disorders, markedly reduced FVC and many associated conditions, is related to a progressive reduction in physical activity. ${ }^{29}$ Depression is an independent risk factor, and regular screening for its occurrence is recommended. ${ }^{30}$

\section{Pulmonary diseases accompanying ILDs}

Each pulmonary disease accompanying ILDs results in an additive effect, causing greater lung function abnormalities and increased mortality compared to each disease separately. A classic example is the coexistence of emphysema and ILDs. In 2005, Cottin et al. described a syndrome of coexistence of upper lobe emphysema and fibrosis of the lower lung lobes. ${ }^{31}$ The incidence of emphysema in ILD cases is estimated at $10 \%$, and primarily concerns smoking males, who are more likely to develop lung cancer and PAH. The frequency of home oxygen therapy is increased in this group. An increase in the incidence of lung cancer is observed in patients with IPF, systemic sclerosis, dermatomyositis, ascites, and asbestosis. ${ }^{24}$

The currently available literature presents 2 different opinions on responses to PR. Some authors estimate that greater improvement is observed in patients whose baseline FVC is higher and whose desaturation after exercise is lower. These authors conclude that it is crucial to introduce PR as early as possible in this group of patients. ${ }^{29}$ Other authors observe that lower 6MWT baseline values result in the greatest increase after PR, suggesting that even in very advanced disease respiratory rehabilitation makes sense. ${ }^{32}$ It seems that both opinions are correct, proving that improvements in ILD patients can be achieved in a variety of diseases with a broad spectrum of progression. ${ }^{33}$

The improvement in ILDs patients after PR persists for up to 6 months, although detailed evaluations are affected by the high percentage of patients who do not complete rehabilitation programs, indicated as $28 \%$ by Ryerson et al. ${ }^{34}$ In a group of 36 patients with ILDs of varying etiology, 16 underwent a 6-month PR program, comprising a total of 60 sessions, and control tests were carried out 1 year after the conclusion. It turned out that improvement in lung function and exercise tolerance are visible even 6 months after finishing a program of this kind. Significant differences between the PR group and the control group were observed after a year, evaluating the 6MWT, muscle strength and maximal workload on a cycle ergometer. ${ }^{35}$ In a group of 48 IPF patients qualified for lung transplantation, 12 weeks of PR were conducted. The patients were characterized by severe lung function impairment (average FVC was $49 \% \pm 13 \%$ of the predicted value and DLCO was $46 \% \pm 17 \%$ ). The improvement was assessed on the basis of the 6MWT and the SF-36 questionnaire. Out of this group, 31 participants successfully completed the PR, and the rest of the respondents did not show any significant differences in the parameters assessed before the start of PR. ${ }^{36}$

In a meta-analysis of 9 PR programs for ILD patients, 8 programs were on an outpatient basis. The average duration was 10 weeks, with 2 sessions per week. Pulmonary rehabilitation complications are rare, mainly involving exercise-induced hypoxemia, ILD-specific symptoms and arrhythmias. Most authors recommend interrupting exercise when $\mathrm{O}_{2}$ saturation drops below $80 \%$. Continuing the exercise in such patients requires oxygen supplementation to achieve saturation above $85 \%$ and even up as high as $90 \% .{ }^{37}$ Oxygen supplementation during PR in patients with ILDs improves exercise tolerance. ${ }^{38}$ 


\section{General rules of qualification for pulmonary rehabilitation in ILDs}

Patients with a broad spectrum of ILDs are qualified for PR, most commonly with IPF, sarcoidosis and postinflammatory pulmonary fibrosis. The qualification procedure should include:

1. a detailed interview;

2. a physical examination in which attention should be paid to the presence of musculoskeletal disorders that may significantly impair the patient's physical activity;

3. cardiac diagnostics, including an evaluation of ischemic changes, arrhythmias and cardiac conduction (especially in patients with sarcoidosis) as well as pulmonary pressure;

4. cardiac safety parameters, including a pulse rate at rest $<120 \mathrm{bpm}$, right ventricular systolic blood pressure (RVSBP) $<40 \mathrm{~mm} \mathrm{Hg}$, ejection fraction $\geq 40 \%$, and no recent ischemic lesions visible on electrocardiography (ECG);

5. elimination of harmful environmental factors like smoking and exposure to allergens;

6. a diagnosis of accompanying diseases;

7. an evaluation of peripheral muscle strength;

8. the ability to live independently.

Pulmonary rehabilitation should be introduced as early as possible, especially for IPF patients. The basic safety parameter is a stable course of the disease. In CTD-ILDs (rheumatoid arthritis, systemic scleroderma), pain and stiffness of the joints can be a problem.

Contraindications to PR include:

1. an unstable course of the disease;

2. fainting after effort during an examination;

3. symptoms of right ventricular cardiovascular failure;

4. an uncontrolled course of accompanying diseases; and

5. any disease that prevents exercise training. ${ }^{20}$

Despite many uncertainties, the current state of knowledge allows PR to be included in standard care in ILD patients in 6-12-month intervals. ${ }^{38}$ Due to the different etiology and prognoses of ILDs, the effects of PR will largely depend on the diagnosis. Especially in diseases with an acute onset, it is advisable to achieve remission or stabilize the disease before starting PR. In IPF, due to the rapid progression of the disease, early qualification for $P R$ is recommended. The positive effects of PR can last up to 6 months. ${ }^{32,39}$

Numerous parameters are used to evaluate the effectiveness of PR in ILDs:

1. changes in the distance in 6MWT;

2. lowest $\mathrm{O}_{2}$ saturation value in the $6 \mathrm{MWT}$ test (nadir $\mathrm{SpO}_{2}$ less exercise-induced oxyhemoglobin desaturation);

3. resting RSVBP; and

4. scores on specific lung disease questionnaires (SGRQ, CRDQ and MRC-BDI).

The $6 \mathrm{MWT}$ in patients with IPF is highly repeatable at short intervals (1-2 weeks) and highly correlated $(\mathrm{r}=0.78)$ with maximal oxygen uptake in the ergospirometry exercise test. The minimum significant difference (MCID) in the 6MWT has been determined as $28 \mathrm{~m} .{ }^{15}$ In another study, based on a study population 822 patients with IPF the estimated MCID was $24-45 \mathrm{~m} .{ }^{16}$ The total distance in the $6 \mathrm{MWT}$ provides an easy evaluation of physical capacity. The $6 \mathrm{MWT}$ score increases with the reduction of fatigue observed after aerobic training. ${ }^{40}$ A gradual decline in the $6 \mathrm{MWT}$ is associated with increased mortality in ILD patients, especially those with IPF. ${ }^{41}$ A meta-analysis of studies performed on small groups of patients (43 in total who underwent PR and 42 controls) showed that the patients with IPF demonstrated less improvement after PR than the patients with other ILDs. In the IPF group, an average increase in the 6MWT immediately after PR was $26.55 \mathrm{~m}$; in the remaining patients with ILDs, it was $38.61 \mathrm{~m}$. This may be related to the initial severity and faster course of the disease in IPF patients. ${ }^{41}$ In a study conducted on a group of 32 patients with IPF, 15 were subjected to a 12-week exercise-based PR program and showed significant improvement in exercise tolerance, functional capacity and FVC. To evaluate exercise tolerance, the cardiopulmonary exercise test was used; after 12 weeks of PR, there was a significant increase in $\mathrm{VO}_{2} \mathrm{max}$. This is an interesting observation, because in patients with other lung diseases, e.g., COPD, there is no improvement in $\mathrm{VO}_{2} \mathrm{max}$ and FVC after PR. Considering that the average survival time of IPF patients is $3-5$ years from diagnosis, improvement in these parameters in a group of IPF patients an average of 2-3 years following their diagnosis shows that even in such a rapidly progressing disease, PR can cause measurable improvement. ${ }^{42}$ Quality of life questionnaires and nadir $\mathrm{SpO}_{2}$ are simple and credible ways to evaluate the patients' response to PR. ${ }^{32}$

The effectiveness of PR in patients with ILD is assessed differently in different publications. Differences are caused by:

1. different patient groups (usually there are better results in ILD groups with mixed etiology);

2. different patient conditions (in patients with IPF, short-term study results are less affected by the rapid disease progression);

3. different PR programs, lasting 12-24 weeks, with varied intensity (usually 2-3 times a week); and

4. varied parameters assessing the effectiveness of PR: exercise tolerance, functional capacity, pulmonary function tests, muscle strength, and health-related quality of life.

\section{Conclusions}

Pulmonary rehabilitation is an important part of comprehensive care for patients with ILDs. The principles of rehabilitation in this group of patients do not differ from the standards of PR for patients with COPD; the major 
differences are due to poorer exercise tolerance and faster development of respiratory failure in patients with ILDs. Exercise-induced desaturation is frequently observed during PR. This is the main source of complications in patients with ILDs, and it occurs more frequently than in COPD patients. Pulmonary rehabilitation centers for ILD patients should be equipped with an oxygen source, a resuscitation kit, a defibrillator, and experienced staff. Early qualification and stabilization of the progression of ILDs and any accompanying diseases can reduce the risk of complications and achieve visible improvement in the patient's health status.

\section{ORCID iDs}

Krzysztof Wytrychowski (1) https://orcid.org/0000-0003-4457-1027 Anna Hans-Wytrychowska (1) https://orcid.org/0000-0003-4842-5262 Paweł Piesiak (1) https://orcid.org/0000-0002-3166-1456 Marta Majewska-Pulsakowska (1) https://orcid.org/0000-0001-6575-0521 Krystyna Rożek-Piechura (1) https://orcid.org/0000-0001-5589-4978

\section{References}

1. Wiatr E. Definicja i klasyfikacje chorób śródmiąższowych płuc. In: Wiatr E, Rowińska-Zakrzewska E, Pirożyński M, eds. Choroby śródmiqż̇szowe płuc. Bielsko-Biała, Poland: Alfa Medica Press; 2012:15-20.

2. Boros P. Zaburzenia czynnościowe $w$ chorobach śródmiąższowych płuc. In: Wiatr E, Rowińska-Zakrzewska E, Pirożyński M, eds. Choroby śródmiqższowe płuc. Bielsko-Biała, Poland: Alfa Medica Press; 2012: 48-61.

3. Duchemann B, Annesi-Maesano I, Jacobe de Naurois C, et al. Prevalence and incidence of interstitial lung diseases in a multi-ethnic county of Greater Paris. Eur Respir J. 2017;50(2). pii: 1602419. doi:10. 1183/13993003.02419-2016

4. Interstitial Lung Diseases - ERS. https://www.erswhitebook.org/ chapters/interstitial-lung-diseases/.

5. Meyer KC. Diagnosis and management of interstitial lung disease. Trans/ Respir Med. 2014;2:4. doi:10.1186/2213-0802-2-4

6. Loveman E, Copley VR, Colquitt J, et al. The clinical effectiveness and cost-effectiveness of treatments for idiopathic pulmonary fibrosis: A systematic review and economic evaluation. Health Technol Assess. 2015;19(20):i-xxiv,1-336.

7. Nici L, Donner C, Wouters E, et al; ATS/ERS Pulmonary Rehabilitation Writing Committee. American Thoracic Society/European Respiratory Society Statement on Pulmonary Rehabilitation. Am J Respir Crit Care Med. 2006;173(12):1390-1413.

8. Spruit MA, Singh SJ, Garvey C, et al; ATS/ERS Task Force on Pulmonary Rehabilitation. An Official American Thoracic Society/European Respiratory Society Statement: Key Concepts and Advances in Pulmonary Rehabilitation. Am J Respir Crit Care Med. 2013;188(8):1011-1027.

9. Rochester CL, Vogiatzis I, Holland AE, et al; ATS/ERS Task Force on Policy in Pulmonary Rehabilitation. An Official American Thoracic Society/European Respiratory Society Policy Statement: Enhancing Implementation, Use, and Delivery of Pulmonary Rehabilitation. Am J Respir Crit Care Med. 2015;192(11):1373-1386.

10. Swigris JJ, Brown KK, Make BJ, Wamboldt FS. Pulmonary rehabilitation in idiopathic pulmonary fibrosis: A call for continued investigation. Respir Med. 2008;102(12):1675-1680.

11. Flaherty KR, Thwaite E, Kazerooni EA, et al. Radiological versus histological diagnosis in UIP and NSIP: Survival implications. Thorax. 2003;58(2):143-148.

12. Holland AE, Hill CJ, Conron M, Munro P, McDonald CF. Short term improvement in exercise capacity and symptoms following exercise training in interstitial lung disease. Thorax. 2008;63(6):549-554.

13. Nishiyama O, Kondoh Y, Kimura T, et al. Effects of pulmonary rehabilitation in patients with idiopathic pulmonary fibrosis. Respirology. 2008;13(3):394-399.

14. Holland A, Hill C. Physical training for interstitial lung disease. Cochrane Database Syst Rev. 2008;4:CD006322.
15. ATS Committee on Proficiency Standards for Clinical Pulmonary Function Laboratories. ATS Statement: Guidelines for the Six-Minute Walk Test. Am J Respir Crit Care Med. 2002;166(1):111-117.

16. du Bois RM, Weycker D, Albera C, et al. Six-minute-walk test in idiopathic pulmonary fibrosis: Test validation and minimal clinically important difference. Am J Respir Crit Care Med. 2011;183(9):1231-1237.

17. Holland AE, Hill CJ, Conron M, Munro P, McDonald CF. Small changes in six-minute walk distance are important in diffuse parenchymal lung disease. Respir Med. 2009;103(10):1430-1435.

18. Bajwah S, Ross JR, Peacock JL, et al. Interventions to improve symptoms and quality of life of patients with fibrotic interstitial lung disease: A systematic review of the literature. Thorax. 2013;68(9):867-879.

19. Swigris JJ, Fairclough DL, Morrison M. Benefits of pulmonary rehabilitation in idiopathic pulmonary fibrosis. Respir Care. 2011;56(6): 783-789.

20. Dowman L, Hill CJ, Holland AE. Pulmonary rehabilitation for interstitial lung disease. Cochrane Database Syst Rev. 2014;10:CD006322.

21. Gomes-Neto M, Silva CM, Ezequiel D, Conceição CS, Saquetto M, Machado AS. Impact of pulmonary rehabilitation on exercise tolerance and quality of life in patients with idiopathic pulmonary fibrosis: A systematic review and meta-analysis. J Cardiopulm Rehabil Prev. 2018;38(5):273-278.

22. Huppmann P, Szczepanski B, Boensch M, et al. Effects of inpatient pulmonary rehabilitation in patients with interstitial lung disease. Eur Respir J. 2013;42(2):444-453.

23. Flaherty KR, Andrei AC, Murray S, et al. Idiopathic pulmonary fibrosis: Prognostic value of changes in physiology and six-minute-walk test. Am J Respir Crit Care Med. 2006;174(7):803-809.

24. Kizer JR, Zisman DA, Blumenthal NP, et al. Association between pulmonary fibrosis and coronary artery disease. Arch Intern Med. 2004; 164(5):551-556.

25. King C, Nathan SD. Identification and treatment of comorbidities in idiopathic pulmonary fibrosis and other fibrotic lung diseases. Curr Opin Pulm Med. 2013;19(5):466-473.

26. Sherner J, Collen J, King CS, Nathan SD. Pulmonary hypertension in idiopathic pulmonary fibrosis: Epidemiology, diagnosis and therapeutic implications. Curr Respir Care Rep. 2012;1(4):233-242.

27. Mereles D, Ehlken N, Kreuscher S, et al. Exercise and respiratory training improve exercise capacity and quality of life in patients with severe chronic pulmonary hypertension. Circulation. 2006;114(14): 1482-1489.

28. de Man FS, Handoko ML, Groepenhoff $H$, et al. Effects of exercise training in patients with idiopathic pulmonary arterial hypertension. Eur Respir J. 2009;34(3):669-675.

29. Fox BD, Kassirer M, Weiss I, et al. Ambulatory rehabilitation improves exercise capacity in patients with pulmonary hypertension. J Card Fail. 2011;17(3):196-200.

30. Holland AE, Fiore JF Jr, Bell EC, et al. Dyspnoea and comorbidity contribute to anxiety and depression in interstitial lung disease. Respirology. 2014;19(8):1215-1221.

31. Cottin V, Nunes H, Brillet P-Y, Delaval P. Combined pulmonary fibrosis and emphysema: A distinct underrecognised entity. Eur Respir J. 2005;26(4):586-593.

32. Holland AE, Hill CJ, Glaspole I, Goh N, Mc-Donald CF. Predictors of benefit following pulmonary rehabilitation for interstitial lung disease. Respir Med. 2012;106(3):429-435.

33. Deniz S, Şahin H, Yalnız E. Does the severity of interstitial lung disease affect the gains from pulmonary rehabilitation? Clin Respir J. 2018;12(6):2141-2150.

34. Ryerson CJ, Cayou C, Topp F, et al. Pulmonary rehabilitation improves long-term outcomes in interstitial lung disease: A prospective cohort study. Respir Med. 2014;108(1):203-210.

35. Perez-Bogerd S, Wuyts W, Barbier V, et al. Short and long-term effects of pulmonary rehabilitation in interstitial lung diseases: A randomised controlled trial. Respir Res. 2018;19(1):182.

36. da Fontoura FF, Berton DC, Watte G, et al. Pulmonary rehabilitation in patients with advanced idiopathic pulmonary fibrosis referred for lung transplantation. J Cardiopulm Rehabil Prev. 2018;38(2): 131-134.

37. Nishiyama $\mathrm{O}$, Kondoh $\mathrm{Y}$, Kimura $\mathrm{T}$, et al. Effects of pulmonary rehabilitation in patients with idiopathic pulmonary fibrosis. Respirology. 2008;13(3):394-399. 
38. Leach RM, Davidson AC, Chinn S, Twort CH, Cameron IR, Bateman NT. Portable liquid oxygen and exercise ability in severe respiratory disability. Thorax. 1992;47(10):781-789.

39. Raghu G, Collard HR, Egan JJ, et al; ATS/ERS/JRS/ALAT Committee on Idiopathic Pulmonary Fibrosis. An Official ATS/ERS/JRS/ALAT Statement. Idiopathic Pulmonary Fibrosis: Evidence-Based Guidelines for Diagnosis and Management. Am J Respir Crit Care Med. 2011; 183(6):788-824.

40. Ferreira A, Garvey C, Connors GL, et al. Pulmonary rehabilitation in interstitial lung disease: Benefits and predictors of response. Chest. 2009;135(2):442-447.
41. Keyser RE, Christensen EJ, Chin LM, Woolstenhulme JG. Changes in fatigability following intense aerobic exercise training in patients with interstitial lung disease. Respir Med. 2015;109(4):517-525.

42. Vainshelboim B, Myers J, Oliveira J, Izhakian S, Unterman A, Kramer MR. Physiological responses and prognostic value of common exercise testing modalities in idiopathic pulmonary fibrosis. $J$ Cardiopulm Rehabil Prev. 2018 Sep 24 [Epub ahead of print]. doi:10.1097/ HCR.0000000000000362 